\title{
Dialysis access as a source of infective endocarditis in dialysis patients
}

\author{
Hee D. Jeon', Kevin B. Lo², Eduardo E. Quintero², Byeori Lee², Asma Gulab², Ruchika Bhargav², \\ Gregg S. Pressman ${ }^{3}$ \\ ${ }^{1}$ Department of Cardiology, Loma Linda University Medical Center, Loma Linda, CA; ${ }^{2}$ Department of Internal Medicine, \\ Albert Einstein Medical Center, Philadelphia, PA; ${ }^{3}$ Department of Cardiology, Albert Einstein Medical Center, \\ Philadelphia PA, USA
}

\begin{abstract}
The incidence of Infective Endocarditis (IE) is higher in dialysis patients compared to the general population. A major risk factor for IE in this group stems from bacterial invasion during repeated vascular access. Previous studies have shown increased risk of bacteremia in patients with indwelling dialysis catheters compared to permanent vascular access. However, association between the development of IE and the type of dialysis access is unclear.
\end{abstract}

We aimed to examine the associated types of intravascular

Correspondence: Hee D. Jeon, Loma Linda University Medical Center, 11234 Anderson Street, Suite 2426, Loma Linda, CA 92354.

Tel.: 1-267-280-6410. Fax: 1-442-212-2307.

E-mail: Heejeon@1lu.edu

Key words: Infective endocarditis; hemodialysis; intravascular catheter; central line-associated bloodstream infection.

Contributions: HDJ was the primary investigator of this study, and was involved in organizing the study design, data collection and interpretation, and writing of the article. KBL, EEQ, BL, AG, RB were each involved in the data collection and interpretation, and review of the manuscript draft. GSP was the faculty mentor of this project, and was involved in organizing the study design, supervising the project progress, and revising the article manuscript.

Conflict of interest: The Authors declare no conflict of interests.

Funding: None.

Further information: This study was presented as poster presentation at American Heart Association Scientific Sessions, November 2019, Philadelphia PA.

Received for publication: 13 July 2020.

Accepted for publication: 28 August 2020.

${ }^{\circ}$ Copyright: the Author(s), 2020

Licensee PAGEPress, Italy

Monaldi Archives for Chest Disease 2020; 90:1505

doi: $10.4081 /$ monaldi.2020.1505

This article is distributed under the terms of the Creative Commons Attribution Noncommercial License (by-nc 4.0) which permits any noncommercial use, distribution, and reproduction in any medium, provided the original author(s) and source are credited. access and route of infection in dialysis patients who were admitted with IE at our center.

All patients admitted to Albert Einstein Medical Center in Philadelphia with a diagnosis of IE who were on chronic hemodialysis were identified from the hospital database for the period of $1 / 1 / 07$ to $12 / 31 / 18$. Modified Duke criteria were used to confirm the diagnosis of IE.

A total of 96 cases were identified. Of those, 57 patients had an indwelling dialysis catheter while the other 39 had permanent dialysis access. In $82 \%$ of patients with dialysis catheters, their dialysis access site was identified as the primary source of infection compared to $30 \%$ in those with permanent dialysis access $(\mathrm{p}<0.001)$. The number of dialysis catheters placed in the preceding 6 months was strongly associated with endocarditis resulting from the dialysis access site $(\mathrm{OR}=3.202, \mathrm{p}=0.025)$.

Dialysis catheters are more likely to serve as the source of infection in dialysis patients developing IE compared to permanent dialysis access. Increased awareness of risk of IE associated with dialysis catheters is warranted.

\section{Introduction}

Patients with End-Stage Renal Disease (ESRD) are at high risk of developing Infective Endocarditis (IE). The risk has been estimated to be 16-18 times the general population [1], with an incidence rate of 267 per 100,000 person-years [2]. Multiple comorbidities associated with ESRD contribute to the increased risk of IE including uremia-related immunosuppression and accelerated valvular calcification [2]. However, the most significant risk factor associated with the development of IE in dialysis patients stems from bacterial invasion on repeated vascular access during dialysis [2].

Bacteremia in dialysis patients has been reported to occur at a rate of 0.7-1.4 episodes per 100 patient-care months [3]. Moreover, the presence of an indwelling dialysis catheter further increases the risk of bacteremia. Retrospective studies have estimated this risk at 1.86 per 1000 days, compared to 0.08 and 0.31 per 1000 days in patients with Arteriovenous Fistulas (AVF) and Arteriovenous Grafts (AVG)[4].

Even though the association between dialysis access and risk of bacteremia is well-established, the association between IE and type of vascular access is less clear. Most of the available studies have small sample sizes showing conflicting results $[5,6]$. This study examined baseline characteristics, risk factors, microbiology, route of infection, outcomes, and predictors of these outcomes in dialysis patients with different types of intravascular access who developed IE. 


\section{Methods}

All patients admitted to Einstein Medical Center Philadelphia (EMCP) with a diagnosis of IE who were on chronic hemodialysis were identified from the hospital database for the period January 1, 2007, to December 31, 2018. Modified Duke criteria were used to confirm the diagnosis of endocarditis. Patients with acute renal failure requiring hemodialysis were excluded. The medical records were reviewed, and data were collected on demographics, comorbidities, microbiology, clinical data, and outcomes. Primary source of infection was identified based on the clinical documentation of the treating healthcare team, imaging findings and review of the microbiology laboratory data including culture of the catheter tip and blood cultures. Acute Physiology and Chronic Health Evaluation (APACHE) II score was calculated for each patient to adjust for the severity of illness in relation to clinical outcomes. The APACHE II score consists of three parts: acute physiological variables (temperature, $\mathrm{pH}$, mean arterial pressure, heart rate, respiratory rate, sodium potassium, creatinine, presence of acute renal failure, hematocrit, WBC count, A-a gradient, Glasgow Coma Scale), age, and chronic health status.

Descriptive statistics were used to analyze demographic and clinical variables. Chi-square analysis was used when comparing categorical variables. Multivariate logistic regression was used to analyze factors associated with surgery and mortality during hospitalization. Logistic regression was also used to explore possible relationships between type of dialysis access and endocarditis among these patients. A p value of less than 0.05 was considered statistically significant. This study was approved by the Institutional Review Board of the Einstein Healthcare Network.

\section{Results}

A total of 640 cases of IE were identified between January $1^{\text {st }}$, 2007, and December 31 $31^{\text {st }}$, 2018. 96 cases were identified in patients with End Stage Kidney Disease (ESKD) patients on chronic dialysis. Of those, 57 had central venous catheter dialysis access as the primary means of dialysis while 39 had a permanent dialysis access such as AVG or AVF. The majority of access sites were in the upper chest and upper extremity. Mean duration of dialysis catheter use was approximately 3 months. Approximately $35 \%$ of patients with temporary dialysis catheter had at least 2 or more catheters placed within the last 6 months prior to the endocarditis (see Table 1). In $82 \%$ of patients with dialysis catheters, their dialysis access site was identified as the primary source of infection compared to $30 \%$ in those with permanent dialysis access $(p<0.001)$ (see Figure 1). More patients in the catheter access group had documented bacteremia preceding IE when compared to the permanet access group (93\% vs 79\%), though this did not reach statistical significance $(\mathrm{p}=0.102)$.

Looking further into the factors associated with infection

Table 1. Demographic and clinical characteristics.

\begin{tabular}{|c|c|c|c|}
\hline & $\begin{array}{c}\text { Temporary/catheter } \\
\text { dialysis access } \\
n=57(\%)\end{array}$ & $\begin{array}{c}\text { Permanent dialysis } \\
\text { access } \\
n=39(\%)\end{array}$ & P value \\
\hline Age (mean \pm SD) & $58.19 \pm 14.37$ & $62.23 \pm 11.84$ & 0.151 \\
\hline Gender Male & $38(67)$ & $25(64)$ & 0.795 \\
\hline $\begin{array}{l}\text { Race } \\
\text { African American } \\
\text { Caucasian } \\
\text { Hispanic } \\
\text { Others }\end{array}$ & $\begin{array}{c}50(88) \\
3(5) \\
3(5) \\
1(2)\end{array}$ & $\begin{array}{l}33(84) \\
3(8) \\
3(8) \\
0\end{array}$ & 0.766 \\
\hline Diabetes & $33(58)$ & $22(56)$ & 0.885 \\
\hline Immunocompromised & $13(23)$ & $10(26)$ & 0.749 \\
\hline Heart failure & $30(53)$ & $24(62)$ & 0.388 \\
\hline Previous valvular disease & $12(21)$ & $6(15)$ & 0.485 \\
\hline $\begin{array}{l}\text { Affected valve with pre-existing valvular disease } \\
\text { Yes, but no prior surgery on the valve } \\
\text { Yes, with prosthetic valve } \\
\text { No }\end{array}$ & $\begin{array}{c}6(11) \\
3(5) \\
48(84)\end{array}$ & $\begin{array}{c}2(5) \\
1(3) \\
36(92)\end{array}$ & 0.500 \\
\hline Prior cardiac surgery & $10(18)$ & $7(18)$ & 0.959 \\
\hline $\begin{array}{l}\text { Type of dialysis access on admission } \\
\text { Temporary trialysis } \\
\text { Permacath } \\
\text { AV fistula } \\
\text { AV graft }\end{array}$ & $\begin{array}{c}1(2) \\
56(98) \\
0 \\
0\end{array}$ & $\begin{array}{c}0 \\
0 \\
16(41) \\
23(59)\end{array}$ & $<0.001$ \\
\hline $\begin{array}{l}\text { Location of access site } \\
\text { Neck } \\
\text { Upper chest } \\
\text { Upper extremity } \\
\text { Groin } \\
\text { Lower extremity }\end{array}$ & $\begin{array}{c}0 \\
54(95) \\
0 \\
3(5) \\
0\end{array}$ & $\begin{array}{c}0 \\
0 \\
38(97) \\
0 \\
1(3)\end{array}$ & $<0.001$ \\
\hline
\end{tabular}

To be continued on next page 
arising from the dialysis catheter, number of previous dialysis catheters in the preceding 6 months was significantly associated with the dialysis catheter being the primary source of infection (OR 3.202, 95\%CI 1.160 to 8.843; $\mathrm{p}=0.025$ ). Meanwhile, age was inversely associated with risk of developing IE from the dialysis access site (OR 0.945 95\%CI 0.902 to 0.989 ; p=0.015) (see Table 2).

The most common causative agent for endocarditis in both the

Table 1. Continued from previous page.

\begin{tabular}{|c|c|c|c|}
\hline & $\begin{array}{c}\text { Temporary/catheter } \\
\text { dialysis access } \\
\mathrm{n}=57(\%)\end{array}$ & $\begin{array}{c}\text { Permanent dialysis } \\
\text { access } \\
\mathrm{n}=39(\%)\end{array}$ & P value \\
\hline Duration of catheter in months & $4.66 \pm 6.12$ & N/A & \\
\hline $\begin{array}{l}\text { \# of catheters } 6 \text { months prior to endocarditis } \\
0 \\
1 \\
2 \\
3 \\
4\end{array}$ & $\begin{array}{c}0 \\
37(65) \\
15(26) \\
4(7) \\
1(2)\end{array}$ & $\begin{array}{l}37(94) \\
1(3) \\
1(3) \\
0 \\
0\end{array}$ & $<0.001$ \\
\hline Dialysis access as cause of infection/endocarditis & $47(84)$ & $12(31)$ & $<0.001$ \\
\hline Preceding bacteremia & $53(93)$ & $31(79)$ & 0.102 \\
\hline $\begin{array}{l}\text { Causative organism } \\
\text { No growth } \\
\text { MSSA } \\
\text { MRSA } \\
\text { Coag. Negative Staph } \\
\text { Strep viridans } \\
\text { Strep bovis } \\
\text { VSE } \\
\text { VRE } \\
\text { Klebsiella } \\
\text { Pseudomonas }\end{array}$ & $\begin{array}{c}2(3) \\
16(28) \\
11(19) \\
9(16) \\
2(3) \\
1(2) \\
11(19) \\
4(8) \\
1(2) \\
0 \\
\end{array}$ & $\begin{array}{c}5(13) \\
12(31) \\
10(26) \\
5(12) \\
3(8) \\
0 \\
3(8) \\
0 \\
0 \\
1(2)\end{array}$ & 0.221 \\
\hline $\begin{array}{l}\text { Valve affected } \\
\text { No vegetation } \\
\text { Aortic } \\
\text { Mitral } \\
\text { Tricuspid } \\
\text { Pulmonic } \\
\text { Catheter } \\
\text { ICD lead } \\
\text { Free wall } \\
\text { Mitral+aortic }\end{array}$ & $\begin{array}{c}1(2) \\
16(28) \\
21(36) \\
9(16) \\
1(2) \\
2(3) \\
1(2) \\
4(8) \\
2(3)\end{array}$ & $\begin{array}{c}2(5) \\
6(16) \\
22(57) \\
7(18) \\
0 \\
0 \\
1(2) \\
0 \\
1(2)\end{array}$ & 0.306 \\
\hline $\begin{array}{l}\text { Vegetation size } \\
\text { No vegetation } \\
<1 \mathrm{~cm} \\
1-1.5 \mathrm{~cm} \\
1.51-2 \mathrm{~cm} \\
>2 \mathrm{~cm} \\
\text { Unknown }\end{array}$ & $\begin{array}{c}0 \\
24(43) \\
10(18) \\
8(14) \\
8(14) \\
6(11)\end{array}$ & $\begin{array}{c}2(5) \\
13(33) \\
9(23) \\
3(8) \\
4(10) \\
8(21)\end{array}$ & 0.271 \\
\hline $\begin{array}{l}\text { Severity of valve regurgitation } \\
\text { None } \\
\text { Mild } \\
\text { Moderate } \\
\text { Severe }\end{array}$ & $\begin{array}{c}9(16) \\
21(36) \\
9(16) \\
18(32)\end{array}$ & $\begin{array}{l}6(15) \\
12(31) \\
12(31) \\
9(23)\end{array}$ & 0.299 \\
\hline Valvular Abscess & $6(11)$ & $2(5)$ & 0.347 \\
\hline Pulmonary edema & $13(23)$ & $12(31)$ & 0.342 \\
\hline Heart block & $6(11)$ & $5(13)$ & 0.729 \\
\hline Pulmonary embolism & $3(5)$ & $3(8)$ & 0.629 \\
\hline Septic emboli & $4(8)$ & $4(10)$ & 0.546 \\
\hline CVA & $4(8)$ & $5(12)$ & 0.338 \\
\hline Underwent surgery & $8(14)$ & $6(15)$ & 0.882 \\
\hline Mortality & $19(33)$ & $10(26)$ & 0.420 \\
\hline APACHE score & $15.75 \pm 2.86$ & $16.05 \pm 2.6$ & 0.605 \\
\hline
\end{tabular}


catheter dialysis access and the permanent dialysis access group was methicillin sensitive $S$. aureus (MSSA), which accounted for over a third of cases. There were more cases of enterococcal endocarditis in the catheter dialysis access group compared to the permanent access group ( $26 \%$ vs $8 \%, \mathrm{p}=0.03$ ) (see Figure 2). There was a total of 4 cases of Vancomycin-Resistant Enterococci (VRE) endocarditis all of which occurred in the catheter dialysis access group.

The mitral and aortic valves were most affected in both the catheter dialysis access group and the permanent dialysis access group accounting for approximately $70 \%$ of cases. However, there were more cases of involvement of unusual areas such as the pulmonic valve, catheter site, ICD leads and free wall involvement in patients with catheter dialysis access even though it did not reach a statistical significance. The majority of vegetations were $<1 \mathrm{~cm}$ in both groups. However, there were numerically more vegetations $>2 \mathrm{~cm}$, more severe valvular regurgitation and higher rates of abscess formation in the temporary dialysis access group though these findings did not reach statistical significance. After multivariate regression, severity of regurgitation (OR 2.251, 1.116 to $4.540, \mathrm{p}=0.023$ ) was associated with higher rates of subsequent surgery (see Table 3 ).

The overall in-hospital mortality rate in this study was $30 \%$, $33.8 \%$ in the temporary dialysis access group $v s 25.6 \%$ in the permanent dialysis access group $(\mathrm{p}=0.396)$. An elevated WBC count on admission, APACHE score, and severity of regurgitation were associated with higher subsequent in-hospital mortality (OR 1.128, p=0.011; OR 1.235, p=0.049; OR 1.717, p=0.047). (see Table 4).

\section{Discussion}

There were four main findings in this study. First, patients with dialysis catheters were significantly more likely to develop IE from the dialysis access compared to patients with permanent vascular access. Second, more frequent catheter changes in the preceding 6 months significantly increased subsequent endocarditis resulting from the dialysis access. Third, enterococcal infection including VRE organism was significantly more common in the catheter dialysis access group than the permanent access group. Lastly, endocarditis carries significant risk of mortality and morbidity in dialysis patients, with the severity of regurgitation, degree of leukocytosis and APACHE score on admission being associated with higher subsequent mortality.

\section{Dialysis access and infection}

Our findings are consistent with previous findings that, among dialysis patients developing bacteremia, the dialysis catheter is the most common primary source of infection compared to an AVF or AVG [7]. The risk of developing IE from a dialysis catheter significantly increased with greater numbers of catheters placed in the 6 months prior to admission. Although tunneled catheters are designed with barriers against infection [8], placement of a central venous catheter still imparts a significant risk for bacteremia. Early after placement, migration of the patient's skin flora, or bacteria on the hands of medical personnel, into the blood stream may cause infection [8]. After 30 days, catheters become infected by internal routes including contamination of the catheter hub, or colonization of the indwelling catheter via hematogenous spread from a distant unrelated infection [8]. A similar trend has been observed with Cardiovascular Implantable Electronic Device (CIED) infections. The risk of CIED infection not only increased with the duration of the device, but also significantly increased with the number of procedures following the initial implantation, demonstrating the significance of infection risk associated with the repeated manipulation [9].

It is notable that younger age was associated with a higher chance of having dialysis access as the primary source of infection. Several previous studies have shown that risk of catheter-related infection in dialysis patients was significantly lower in the elderly population. Murea et al. [10] demonstrated that elderly patients (>75 years) on dialysis via indwelling catheter had a lower risk of CRBSI compared to non-elderly subjects, with the risk of CRBSI decreasing by $10 \%$ for each 5 -year increase in patient age. This finding was also present in a study by Soi et al., which observed a significantly decreased rate of CRBSI among elderly dialysis patients [11]. Potential mechanisms that could explain this finding include lower rates of nasal $S$. aureus carriage [12] and skin infection in the elderly, as well as lower functional status leading to lower chance of common catheter complications seen in active individuals i.e. catheter dislodgement [11].

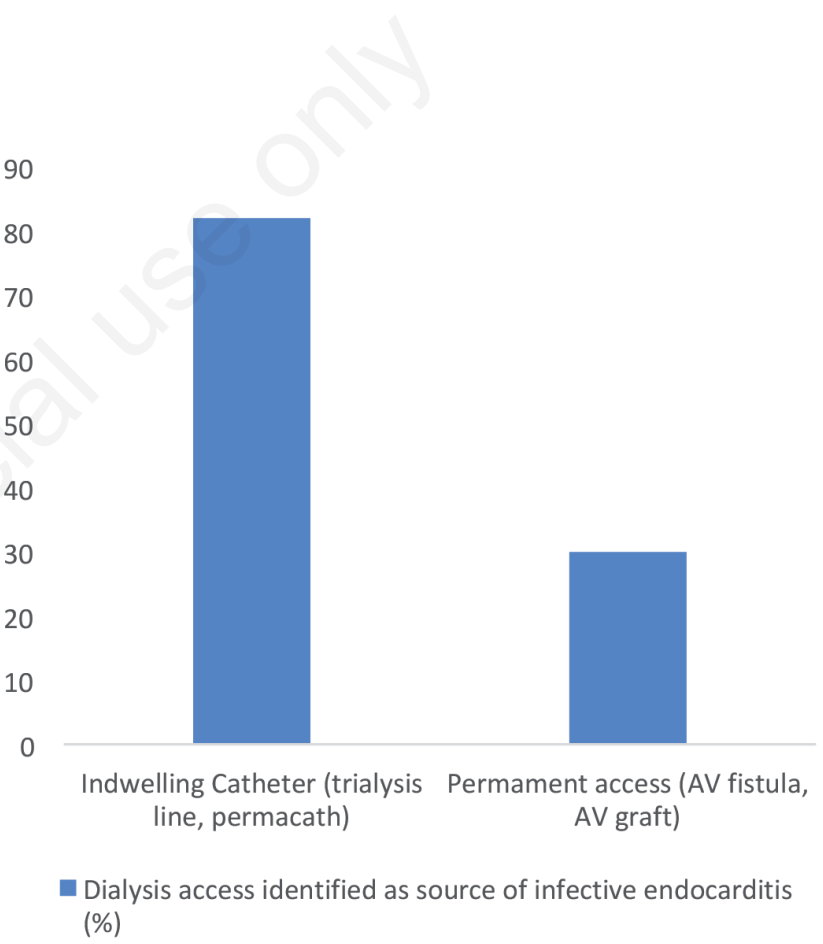

Figure 1. Dialysis access identified as source of infective endocarditis.

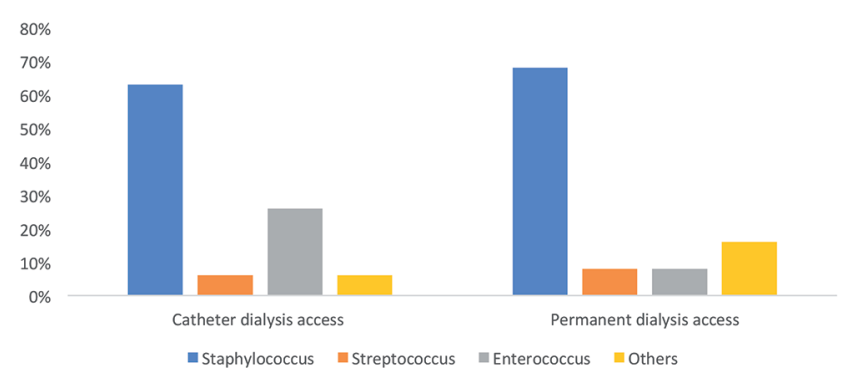

Figure 2. Causative organisms for infective endocarditis by dialysis access. 


\section{Microbiology}

The most common causative agent for endocarditis in both groups was methicillin sensitive $S$. aureus which accounted for over a third of cases. This is consistent with prior studies showing that staphylococcal species is the most common organisms causing IE in dialysis patients [2,13]. In our study, infection with nonstaphylococcal organisms led to less subsequent surgeries compared to infection with staphylococcal microorganisms. This is likely related to the fact that staphylococcal endocarditis is associated with higher rates of complications including septic shock, embolic events, and annular abscess, which often require surgical therapy [14]. Infection by enterococcal species was significantly more common in the catheter dialysis access group compared to the permanent access group ( $26 \%$ vs $8 \%, \mathrm{p}=0.03)$. It was also notable that all 4 cases of VRE infection occurred in the catheter dialysis access group. Enterococcus faecalis is a highly virulent organism with a high propensity for IE; it has been previously demonstrated that the prevalence of IE is estimated to be $26 \%$ in patients with enterococcal bacteremia from all causes [15]. Increased incidence of enterococcal infection with dialysis catheters is not surprising as enteroccocal species are the second most frequent isolates for Central Line Associated Bloodstream Infection (CLABSI) in the USA according to the National Healthcare Safety Network [16].

Table 2. Multivariate regression showing factors associated with dialysis access as a source of endocarditis.

\begin{tabular}{lccc} 
Covariates & Odd ratio & $95 \%$ CI & P value \\
Age & 0.945 & 0.902 to 0.989 & 0.015 \\
Gender & 0.714 & 0.213 to 2.393 & 0.585 \\
\hline Race & 0.571 & 0.227 to 1.435 & 0.233 \\
Diabetes & 2.687 & 0.809 to 8.927 & 0.107 \\
\hline Immunocompromised & 0.672 & 0.174 to 2.598 & 0.564 \\
Prior cardiac surgery & 0.163 & 0.035 to 0.753 & 0.020 \\
\hline Location of access site & 0.419 & 0.139 to 1.261 & 0.122 \\
Duration of catheter & 1.090 & 0.931 to 1.277 & 0.283 \\
\hline Number of dialysis catheters prior to infective endocarditis & 3.202 & 1.160 to 8.843 & 0.025 \\
\hline
\end{tabular}

Table 3. Multivariate regression showing factors associated with surgery.

\begin{tabular}{lccc}
\hline Covariates & Odd ratio & $95 \%$ CI & P value \\
Age & 1.021 & 0.967 to 1.077 & 0.458 \\
Gender & 1.970 & 0.394 to 9.858 & 0.409 \\
\hline Race & 0.854 & 0.264 to 2.757 & 0.792 \\
Diabetes & 0.511 & 0.131 to 1.993 & 0.334 \\
\hline Prior cardiac surgery & 0.249 & 0.025 to 2.507 & 0.238 \\
Severity of regurgitation & 2.251 & 1.116 to 4.540 & 0.023 \\
\hline Abscess & 1.094 & 0.145 to 8.243 & 0.930 \\
Valve affected & 0.814 & 0.498 to 1.332 & 0.413 \\
\hline Staph vs Non Staph & 1.144 & 0.289 to 4.526 & 0.848 \\
\hline
\end{tabular}

Table 4. Multivariate regression showing factors associated with mortality.

\begin{tabular}{lccc} 
Covariates & Odd ratio & $95 \%$ CI & P value \\
Age & 0.971 & 0.930 to 1.015 & 0.190 \\
Gender & 0.827 & 0.286 to 2.394 & 0.726 \\
\hline Race & 0.507 & 0.145 to 1.774 & 0.288 \\
Diabetes & 0.764 & 0.264 to 2.207 & 0.619 \\
\hline Prior cardiac surgery & 0.780 & 0.199 to 3.048 & 0.721 \\
Valve affected & 1.087 & 0.805 to 1.467 & 1.087 \\
\hline Severity of regurgitation & 1.717 & 1.008 to 2.927 & 0.047 \\
WBC on admission & 1.128 & 1.028 to 1.238 & 0.011 \\
\hline APACHE score & 1.235 & 1.001 to 1.523 & 0.049 \\
Underwent surgery & 0.439 & 0.0086 to 2.230 & 0.321 \\
\hline
\end{tabular}




\section{Mortality and subsequent surgery}

In our study, In-hospital mortality was $30 \%$. In a large series of 13,130 hemodialysis patients with IE, Shroff et al. found an inhospital mortality rate of $23.5 \%$ [17], whereas other smaller observational studies have reported in-hospital mortality ranging between $22 \sim 56 \%[18,19,20]$. Even though it did not reach statistical significance $(\mathrm{p}=0.420)$, mortality was numerically higher in the catheter group compared to the permanent access group. There were also more vegetations with size $>2 \mathrm{~cm}$, more severe valvular regurgitation and higher rates of abscess formation in the temporary dialysis access group. To date, no large-sample studies have shown a difference in mortality associated with the presence of a temporary dialysis catheter in IE. In our study, a high white blood cell count on admission and higher Acute Physiologic and Chronic Health Evaluation II (APACHE II) score were associated with higher subsequent hospital mortality. A high APACHE II score is a well-established predictor of increased mortality in IE in the general population $[18,19]$. A study on dialysis patients with $S$. aureus IE showed that APACHE II score was higher in patients with dialysis catheters compared to permanent access, however its association with increased mortality has not been observed [20]. Severity of regurgitation was associated with both higher rate of undergoing surgery and mortality (see Table 3). Higher rate of surgery associated with severity of regurgitation is expected, as acute heart failure due to valvular regurgitation is a widely accepted indication for surgery [21]. Kiefer et al. have demonstrated that severity of heart failure was the strongest predictor of in-hospital and 1 -year mortality in IE, and surgical treatment of the valvular regurgitation significantly reduced this risk across the spectrum of heart failure severity [22]. In our study we observed decreased mortality in patients undergoing surgery that was not statistically significant, likely due to the small size sample.

\section{Study limitations}

This study is limited by the retrospective nature of this analysis and the small sample size. Some potential confounding factors might not have been accounted for including the type of antibiotic used, the presence of IV drug use, the response to the antibiotic used, including time to clear cultures, and the inherent differences in technique in dialysis line or access placement. It should be noted that the majority of patients in our sample also had their dialysis access on the upper chest or upper extremities. Data on the primary source of infection was obtained from clinicians' documentation, which were largely based on clinical judgement with the use of the Modified Duke criteria. It is always possible that the presence of an occult infection aside from the dialysis access infection might have been present and that the dialysis access infection might have just been seeded by bacteria from a remote alternative site.

\section{Conclusions}

Dialysis catheters are more likely to serve as the source of infection in dialysis patients developing IE compared to permanent dialysis access. Increased awareness of risk of IE associated with dialysis catheters is warranted.

\section{References}

1. Nucifora G, Badano L, Viale P, et al. Infective endocarditis in chronic haemodialysis patients: an increasing clinical challenge. Eur Heart J 2007;28:2307-12.

2. Bhatia N, Sahil A, Aakash G, et al. Trends and outcomes of infective endocarditis in patients on dialysis. Clin Cardiol 2017;40:423-9.

3. Maraj S, Larry EJ, Rajiv M, et al. Bacteremia and infective endocarditis in patients on hemodialysis. Am J Med Sci 2004;327:242-9.

4. Zhang J, Renee AB, Heena SS, et al. Organism-specific bacteremia by hemodialysis access. Clin Nephrol 2016; 86:141-6.

5. Chaudry MS, Nicholas C, Gunnar HG, et al. Risk of infective endocarditis in patients with end stage renal disease. Clin J Am Soc Nephro 2017;12:1814-22.

6. Doulton T, Nikhant S, Hugh SC, Silke S, et al. Infective endocarditis in dialysis patients: new challenges and old. Kidney Int 2003;64:720-7.

7. Suzuki M, Nobuhiko S, Motonobu N, et al. Bacteremia in hemodialysis patients. World J Nephrol 2016;5:489.

8. Santoro D, Benedetto F, Mondello P, et al. Vascular access for hemodialysis: current perspectives. Int J Nephrol Renovasc Dis 2014;7:281.

9. Dai M, Cai C, Vaibhav V, et al. Trends of cardiovascular implantable electronic device Infection in 3 decades. JACC Clin Electrophysiol 2019;5:1071-80.

10. Murea M, Kimone MJ, Greg BR, et al. Risk of catheter-related bloodstream infection in elderly patients on hemodialysis. Clin J Am Soc Nephro 2014;9:764-70.

11. Soi V, Lalathaksha K, Jerry Y, et al. Prevention of catheterrelated bloodstream infections in patients on hemodialysis: challenges and management strategies. Int J Nephrol Renovasc Dis 2016;9:95-103.

12. Jean G, Charra B, Chazot C, et al. Risk factor analysis for long-term tunneled dialysis catheter-related bacteremias. Nephron 2002;91:399-405.

13. Miller LM, Edward C, Christine D, et al. Hemodialysis tunneled catheter-related infections. Can J Kidney Health Dis 2016;3:2054358116669129.

14. Han SM, Sorabella RA, Vasan S, et al. Influence of staphylococcus aureus on outcomes after valvular surgery for infective endocarditis. J Cardiothorac Surg 2017;12:57.

15. Dahl A, Iversen K, Tonder N, et al. Prevalence of infective endocarditis in enterococcus faecalis bacteremia. J Am Coll Cardiol 2019;74:193-201.

16. Haddadin Y, Annamaraju P, Regunath H. Central line associated blood stream infections (CLABSI). StatPearls 2019. Available from: https:/www.ncbi.nlm.nih.gov/books/NBK430891/. Accessed 2020 Jul 12.

17. Shroff GR, Charles AH, Jennie ZM, et al. Long-term survival of dialysis patients with bacterial endocarditis in the United States. Am J Kidney Dis 2004;44:1077-82.

18. Bashir MA, Davis TA, Bhama JK, et al. Predicting outcomes and mortality for critically ill patients with infective endocarditis. J Am Coll Surgeons 2017;225.

19. Chu VH, Cabell CH, Benjamin DK, et al. Early predictors of in-hospital death in infective endocarditis. Circulation 2004;109:1745-49.

20. Inrig JK, Reed SD, Szczech LA, et al. Relationship between clinical outcomes and vascular access type among hemodialy- 
sis patients with staphylococcus aureus bacteremia. Clin J Am Soc Nephro 2006;1:518-24.

21. Nishimura RA, Otto CM, Bonow RO, et al. 2017 AHA/ACC Focused update of the 2014 AHA/ACC guideline for the management of patients with valvular heart disease. Circulation
2017;135:1159-95.

22. Kiefer T, Park L, Tribouilloy C, et al. Association between valvular surgery and mortality among patients with infective endocarditis complicated by heart failure. JAMA 2011; 306:2239-47. 